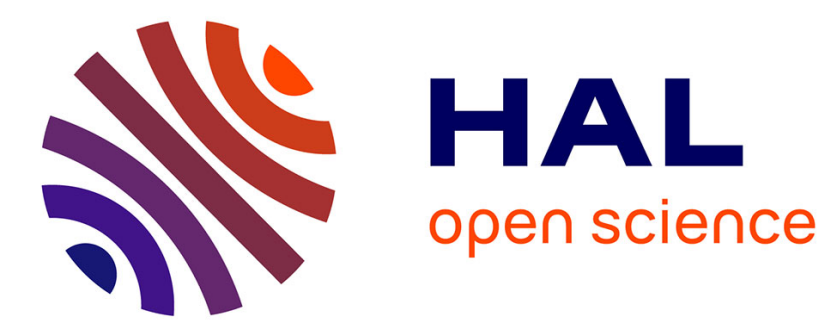

\title{
Adapted kriging to predict the intensity of partially observed point process data.
}

Edith Gabriel, Florent Bonneu, Pascal P. Monestiez, Joel J. Chadoeuf

\section{To cite this version:}

Edith Gabriel, Florent Bonneu, Pascal P. Monestiez, Joel J. Chadoeuf. Adapted kriging to predict the intensity of partially observed point process data.. Spatial Statistics, 2016, 18 (Part A November 2016), pp.54-71. 10.1016/j.spasta.2016.05.004 . hal-01334906

\section{HAL Id: hal-01334906 https://hal.science/hal-01334906}

Submitted on 27 May 2020

HAL is a multi-disciplinary open access archive for the deposit and dissemination of scientific research documents, whether they are published or not. The documents may come from teaching and research institutions in France or abroad, or from public or private research centers.
L'archive ouverte pluridisciplinaire HAL, est destinée au dépôt et à la diffusion de documents scientifiques de niveau recherche, publiés ou non, émanant des établissements d'enseignement et de recherche français ou étrangers, des laboratoires publics ou privés. 


\title{
Adapted kriging to predict the intensity of partially observed point process data
}

\author{
Edith Gabriel $^{\mathrm{a}, \mathrm{b}, \mathrm{d}, *}$, Florent Bonneu ${ }^{\mathrm{a}}$, Pascal Monestiez ${ }^{\mathrm{b}, \mathrm{d}}$, \\ Joël Chadœuf ${ }^{\mathrm{c}}$ \\ a Avignon University, LMA EA 2151, 84000 Avignon, France \\ ${ }^{\mathrm{b}}$ INRA, BioSP, 84000 Avignon, France \\ ${ }^{\mathrm{c}}$ INRA, Statistics, GAFL, UR 1052, 84000 Avignon, France \\ ${ }^{\mathrm{d}}$ Zone Atelier Plaine E Val de Sèvre, CEBC-CNRS, 79360 Villiers-en-Bois, France
}

\section{A R T I C L E I N F O}

\section{Article history:}

Received 15 December 2015

Accepted 25 May 2016

Available online $\mathrm{xxxx}$

\section{Keywords:}

Intensity estimation

Point process

Prediction

Spatial statistics

\begin{abstract}
A B S T R A C T
We consider a stationary and isotropic spatial point process whose realization is observed within a large window. We assume it to be driven by a stationary random field $U$. In order to predict the local intensity of the point process, $\lambda(x \mid U)$, we propose to define the first- and second-order characteristics of a random field, defined as the regularized counting process, from the ones of the point process and to interpolate the local intensity by using a kriging adapted to the regularized process.
\end{abstract}

(c) 2016 Elsevier B.V. All rights reserved.

\section{Introduction}

In many projects the study window is too large to extensively map the intensity of the point process of interest since observation methods may be available at a much smaller scale only. That is for instance the case when studying the spatial repartition of a bird species at a national scale, while the observations are made in windows of few hectares. The intensity must then be estimated from data issued out of samples spread in the study window, and hence, from a partial realization of the point process in this window.

In the following, we consider a stationary and isotropic point process, $\Phi$, which we assume to be driven by a stationary random field, $U$. We define the local intensity of $\Phi$ by its intensity conditional to

\footnotetext{
* Corresponding author at: Avignon University, LMA EA 2151, 84000 Avignon, France.

E-mail address: edith.gabriel@univ-avignon.fr (E. Gabriel).
} 


\section{ARTICLE IN PRESS}

the random field $U$. We denote it $\lambda(x \mid U)$. A simple example of such a process $\Phi$ is the Thomas process which is a Poisson cluster process where the cluster centers (parents) are assumed to be Poisson and the offsprings are normally distributed around the parent point. This process is stationary and the local intensity corresponds to the intensity of the inhomogeneous Poisson process of offsprings, i.e. the conditional intensity given the parent process. We will refer to the estimation of the local intensity when we want to the know it at point locations lying in the observation window of the point process, and to its prediction when point locations are outside the observation window.

Usually, when estimating a non-constant intensity, we observe the full point pattern within a window and we want to know its local changes over a given mesh. This issue has been addressed in several ways: kernel smoothing, see Silverman (1986) and Guan (2008) in presence of covariates, and van Lieshout (2012) for a general class of weight function estimators that encompasses both kernel and tessellation based estimators; or parametric methods; see for instance (Illian et al., 2008) for a review. A recurrent and remaining question in these approaches is which bandwidth/mesh should we use? This has been addressed by using cross-validation (Härdle, 1991) or double kernel (Devroye, 1989).

In contrast to the previous methods which look at the intensity changes inside the observation window, our main interest lies in predicting the intensity outside the observation window, all the more when it is not connected as it frequently happens when sampling in plant ecology. To predict the intensity we could use (Tscheschel and Stoyan, 2006)'s reconstruction method based on the first- and second-order characteristics of the point process. Once the empirical point pattern predicted within a given window, one can get the intensity by kernel smoothing. As it is a simulation-based method, it requires long computation times, especially when the prediction window is large and/or the point process is complex. As alternative method, few authors model the point pattern by a point process with the intensity driven by a stationary random field. In Diggle and Ribeiro (2007) and Diggle et al. (2013), the approach is heavily based on a complete modeling and considers a log-Gaussian model. The parameter estimation, the intensity estimation and its prediction outside the observation window are obtained using a Bayesian framework. The method developed in Monestiez et al. (2006) and Bellier et al. (2013) is close to classical geostatistics. Basically, it consists of counting the number of points within some grid cells, computing the related empirical variogram and theoretically relating it to the one obtained from the random field driving the intensity. Then, the variogram is fitted and kriging is used to predict the intensity. Its advantage is that the estimation is only based on its first- and secondorder moments so that the model does not need to be fully specified. While this approach requires less hypotheses, the model remains constrained within the class of Cox processes. Moreover, the mesh size is arbitrary defined. van Lieshout and Baddeley (2001) developed, for a wider class of parametric models, a Bayesian approach for extrapolating and interpolating clustered point patterns.

Here, we propose to interpolate the local intensity by an adapted kriging, where the kriging weights depend on the local structure of the point process. Hence, our method uses all the data to locally predict at a given point, which it is not the case of most of kernel methods. It also uses the information at a fine scale of the point process, which it is not the case in geostatistical approaches. Furthermore, it does not require a specific model but only (an estimation of) the first- and second-order characteristics of the point process.

In Section 2 we define the regularized process as a random field of point counts on grid cells and we link up the mean and variogram of this random field to the intensity and pair correlation function of the point process. The kriging weights, the related interpolator and its properties are presented in Section 3 as well as the optimal mesh of the interpolation grid. In Section 4 we use our kriging interpolator to estimate and predict the intensity of Montagu's Harriers' nest locations in a region of France. In Section 5, we discuss the influence of the mesh and the rate and shape of unobserved areas on the statistical properties of our kriging interpolator from numerical results.

\section{Linking up characteristics of two theories}

\subsection{About geostatistics}

For any real valued random field $Z(x), x \in \mathbb{R}^{2}$, the first-order characteristic is the mean value function: $\mathbb{E}[Z(x)]=m(x)$ and the second-order characteristics are classically described in 


\section{ARTICLE IN PRESS}

geostatistics (Matheron, 1962, 1963) by the (semi)-variogram, i.e. the mean squared difference at lag $r: \gamma(r)=\frac{1}{2} \mathbb{E}\left[(Z(x)-Z(x+r))^{2}\right]$. For a stationary and isotropic random field, we have

$$
\begin{aligned}
\mathbb{E}[Z(x)] & =m, \\
\gamma(r) & =\sigma^{2}-\operatorname{Cov}(Z(x), Z(x+h)),
\end{aligned}
$$

where $\sigma^{2}$ is the field variance and $\operatorname{Cov}(Z(x), Z(x+r))$ is the auto-covariance of the random field.

We can interpolate the value $Z\left(x_{0}\right)$ at the unsampled location $x_{0}$ by using the best linear unbiased predictor, so-called kriging interpolator: $\widehat{Z}\left(x_{0}\right)=\mu^{T} z$, where $z=\left\{Z\left(x_{i}\right)\right\}_{i=1, \ldots, n}$ is the observation vector of the random field and $\mu$ is the $n$-vector of weights. In the case of ordinary kriging (Cressie, 1993), which will be of interest here since the mean value of the random field will be unknown, we have

$$
\mu=C^{-1} C_{o}+\frac{1-\mathbf{1}^{T} C^{-1} C_{o}}{\mathbf{1}^{T} C^{-1} \mathbf{1}} C^{-1} \mathbf{1},
$$

where $C=\left\{\operatorname{Cov}\left(Z\left(x_{i}\right), Z\left(x_{j}\right)\right)\right\}_{i, j=1, \ldots, n}$ is the covariance matrix between the observations, $C_{o}=$ $\left\{\operatorname{Cov}\left(Z\left(x_{i}\right), Z\left(x_{0}\right)\right)\right\}_{i=1, \ldots, n}$ is the covariance vector between the observations and $Z\left(x_{0}\right)$ and $\mathbf{1}$ is the $n$-vector of 1 (see e.g. Cressie, 1993, Wackernagel, 2003).

\subsection{About point processes}

Let $\Phi$ be a stationary and isotropic point process defined in $\mathbb{R}^{2}$ and $B$ a Borel set centered at 0 . Following the notations in Chiu et al. (2013), a realization of $\Phi$ within a window $S_{o b s}$ will be denoted by $\Phi_{S_{o b s}}$ and the random counting measure for a Borel set $B$ by $\Phi(B)$.

The first- and second-order characteristics of $\Phi$ are described through its intensity $\lambda$ and the Ripley's $K$-function or the pair correlation function $g$ :

$$
\begin{aligned}
\lambda & =\frac{\mathbb{E}\left[\Phi\left(S_{o b s}\right)\right]}{v\left(S_{o b s}\right)}, \\
K^{*}(r) & =\frac{1}{\lambda} \mathbb{E}[\Phi(b(0, r))-1 \mid 0 \in \Phi], \\
g(r) & =\frac{1}{2 \pi r} \frac{\partial K^{*}(r)}{\partial r},
\end{aligned}
$$

where $v\left(S_{o b s}\right)$ is the area of $S_{o b s}$ and $b(0, r)$ is the disc centered at 0 , with radius $r$. The intensity $\lambda$ is thus the expected number of points per unit area, $\lambda K^{*}(r)$ is the mean number of points in a circle of radius $r$ centered at a typical point of the point process, whereas $g(r)$ measures how $K^{*}$ changes with $r$. See for instance (Chiu et al., 2013) for a review about the theory of point processes.

Lemma 2.1. Let $\Phi$ be a point process with intensity $\lambda$ and $B, D$ two Borel sets. Then,

1. If $v(B), v(D) \rightarrow 0$, then $\mathbb{P}[\{\Phi(B)=1\} \cap\{\Phi(D)=1\}]=\lambda \nu(B \cap D)+\lambda^{2} \int_{B \times D} g(x-y) d x d y+$ $o(v(B \cup D))$,

2. $\mathbb{E}\left[\Phi^{2}(B)\right]=\lambda \nu(B)+\lambda^{2} \int_{B \times B} g(x-y) d x d y$,

3. $\operatorname{Var}(\Phi(B))=\lambda v(B)+\lambda^{2} \int_{B \times B}(g(x-y)-1) d x d y$,

4. If $B \cap D=\emptyset$, then $\operatorname{Cov}(\Phi(B), \Phi(D))=\lambda^{2} \int_{B \times D}(g(x-y)-1) d x d y$.

The proof of 1. is given in Appendix A. Decompositions 2.-4. can be found in Chiu et al. (2013).

\subsection{Linking up}

In our context, data are defined as informative point locations (the realization of the point process $\Phi)$ while the geostatistical calculations (kriging) need to be carried out over the values of a random field $Z$ observed at several sampling locations, grid cell centers for example. Thus, we must regularize our process over a compact set (Zhang et al., 2014). This consists in defining $Z(x)$ by the count of 


\section{ARTICLE IN PRESS}

the point process over the grid cell $B$ centered at $x \in \mathbb{R}^{2}$ i.e. $Z(x)=\Phi(B \oplus x)$. Such a random field is of interest in our case as we want to estimate a non-constant intensity, classically defined by $\lim _{v(B) \rightarrow 0} \mathbb{E}\left[\frac{\Phi(B \oplus x)}{v(B)}\right]$.

From the first- and second-order moments defined in the previous sections, we can link up the characteristics of the point process $\Phi$ to the ones of the random field of point counts $Z$. Because of the stationary assumption it can also be related to the auto-covariance function (Eq. (1)), thus in the following we shall consider the latter.

Proposition 2.2. For the count random field defined by $\Phi(B \oplus x)$, where $B$ is a given Borel set, we have:

1. $m=\lambda v(B)$,

2. For $B$ and $D$ two regularization blocks, $B=D+r, B_{D}=B \backslash D, D_{B}=D \backslash B$,

$$
\begin{aligned}
2 \gamma(r)= & \lambda\left(v\left(B_{D}\right)+v\left(D_{B}\right)\right)+\lambda^{2}\left(\int_{B_{D} \times B_{D}} g(x-y) d x d y\right. \\
& \left.+\int_{D_{B} \times D_{B}} g(x-y) d x d y-2 \int_{B_{D} \times D_{B}} g(x-y) d x d y\right) .
\end{aligned}
$$

3. If $B=D+r$, then for $v(B)=v(D) \rightarrow 0$

$$
\operatorname{Cov}(\Phi(B), \Phi(D)) \approx \lambda \nu(B)\left(\mathbb{I}_{\{B=D\}}+\lambda v(B)(g(r)-1)\right) .
$$

The proof of Proposition 2.2 is straightforward from Lemma 2.1 and from the approximation $\mathbb{P}[\{\Phi(B)=1\} \cap\{\Phi(D)=1\}] \approx \lambda^{2} v(B) v(D) g(r)$ (see Appendix B).

\section{Adapted kriging for point processes}

In what follows, we consider that the stationary and isotropic point process, $\Phi$, is driven by a stationary random field, $U$. We want to interpolate the local intensity of $\Phi, \lambda(x \mid U), x \in S \subset \mathbb{R}^{2}$, i.e. its intensity conditional to the random field $U$, from its realization within an observation window $S_{o b s}$. Hence, we use the relation between point processes and geostatistics (Section 2.3) and approximate the point process by the counting process within a grid of elementary cell $B$.

For sake of clarity, in the following we denote by $S$ the region of interest so that $S_{\text {unobs }}$ define the complementary of $S_{o b s}$ within $S$. We consider a regular grid superimposed on $S$ with a square-mesh. We denote by $B$ an elementary square centered at $0, B_{i}=x_{i} \oplus B$ the elementary square centered at $x_{i}$ such that $B_{i} \cap B_{j}=\emptyset$, and $n$ (resp. $n_{o b s}$ ) the number of grid cell centers lying in $S$ (resp. $S_{o b s}$ ).

\subsection{Defining the interpolator}

According to the classical geostatistical method defined in Section 2, the kriging interpolator of the local intensity at $x_{0} \in S, \lambda\left(x_{0} \mid U\right)$, should be written as

$$
\mu^{T}\left(\lambda\left(x_{1} \mid U\right), \ldots, \lambda\left(x_{n_{o b s}} \mid U\right)\right),
$$

for some well-chosen kriging weights $\mu$ where $x_{i}, i=1, \ldots, n_{o b s}$ correspond to data sample locations, i.e. here to the cell centers of $S_{o b s}$. Note that in our case we cannot observe the local intensity at $x_{i}$, thus we can estimate it by $\frac{\Phi\left(B_{i}\right)}{v(B)}$. Furthermore because of the cell-point relation, we cannot have an exact interpolation of the local intensity.

Proposition 3.1. Given the elementary square $B$, the interpolator at $x_{0}$ defined by

$$
\widehat{\lambda}\left(x_{0} \mid U\right)=\sum_{x_{i} \in S_{o b s}} \mu_{i} \frac{\Phi\left(B_{i}\right)}{v(B)},
$$




\section{ARTICLE IN PRESS}

where $\mu=\left(\mu_{1}, \ldots, \mu_{n_{o b s}}\right)=C^{-1} C_{o}+\frac{1-\mathbf{1}^{T} C^{-1} C_{o}}{\mathbf{1}^{T} C^{-1} \mathbf{1}} C^{-1} \mathbf{1}$, is the best linear unbiased predictor (BLUP) of $\frac{\Phi\left(B_{0}\right)}{v(B)}$ and the asymptotically BLUP of $\lambda\left(x_{0} \mid U\right)$.

The weights depend on

- the covariance matrix $C=\lambda v(B) \boldsymbol{I}+\lambda^{2} v^{2}(B)(G-1)$,

where $G=\left\{g_{i j}\right\}_{i, j=1, \ldots, n_{o b s}}$, with $g_{i j}=\frac{1}{v^{2}(B)} \int_{B \times B} g\left(x_{i}-x_{j}+u-v\right) d u d v$, and $\mathbf{I}$ is the $n_{o b s} \times n_{o b s}$-identity matrix,

- the covariance vector $C_{0}=\lambda v(B) \mathbb{I}_{x_{0}}+\lambda^{2} v^{2}(B)\left(G_{0}-1\right)$,

where $G_{o}=\left\{g_{i o}\right\}_{i=1, \ldots, n_{o b s}}$, and $\mathbb{I}_{x_{0}}$ is the $n_{o b s}$-vector with zero values and one term equals to one where $x_{0}=x_{i}$ (which only happens in estimation).

When the distance between $x_{0}$ and $x_{i}$, for all $i$, is larger than the range of interaction, the predicted value tends to $\lambda$ if $x_{o} \notin S_{o b s}$ and the estimated value tends to 0 if $x_{o} \in S_{o b s}$.

Proof. At the scale of $B$, the kriging weights such that $\widehat{\lambda}\left(x_{0} \mid U\right)$ is a BLUP of $\frac{\Phi\left(B_{0}\right)}{v(B)}$ are given by the ordinary kriging equations (Cressie, 1993; Chilès and Delfiner, 2012).

At a finer scale we have that $\mathbb{E}\left[\frac{\Phi(B \oplus x)}{v(B)}\right]$ tends to $\lambda(x \mid U)$ when $v(B)$ tends to 0 (as $\lambda(x \mid U)$ is assumed to be continuous). Thus we propose to interpolate $\lambda\left(x_{0} \mid U\right)$ by using $\widehat{\lambda}\left(x_{o} \mid U\right)=\sum_{x_{i} \in S_{o b s}} \mu_{i} \frac{\Phi\left(B_{i}\right)}{v(B)}$, with the constraint $\sum_{i=1}^{n_{o b s}} \mu_{i}=1$. Minimizing the error variance $\operatorname{Var}\left(\widehat{\lambda}\left(x_{0} \mid U\right)-\lambda\left(x_{0} \mid U\right)\right)$ under this constraint and using Eq. (6) lead to the following kriging weights:

$$
\mu=v(B) C^{-1} \widetilde{C}_{o}+\frac{1-v(B) \mathbf{1}^{T} C^{-1} \widetilde{C}_{o}}{\mathbf{1}^{T} C^{-1} \mathbf{1}} C^{-1} \mathbf{1},
$$

where $\widetilde{C}_{o}=\left\{\operatorname{Cov}\left(\Phi\left(B_{i}\right), \lambda\left(x_{o} \mid U\right)\right)\right\}_{i=1, \ldots, n_{o b s}}$.

To get $\operatorname{Cov}\left(\Phi\left(B_{i}\right), \lambda\left(x_{0} \mid U\right)\right)$, note that, for $\lambda_{\Phi_{B}}(x \mid U)$ denoting the local intensity of the point process $\Phi$ given its realization in $B$ :

$$
\mathbb{E}\left[\Phi\left(B_{o}\right) \mid \Phi_{B_{i}}\right]=\int_{B_{0}} \lambda_{\Phi_{B_{i}}}(x \mid U) \mathrm{d} x=\lambda_{\Phi_{B_{i}}}\left(x_{o} \mid U\right) v(B)+o(v(B))
$$

and $\lambda_{\Phi_{B_{i}}}\left(x_{o} \mid U\right)=\mathbb{E}\left[\lambda_{\Phi_{S_{o b s}}}\left(x_{o} \mid U\right) \mid \Phi_{B_{i}}\right]$. Thus, we have

- for $x_{i} \neq x_{0}$,

$$
\begin{aligned}
\mathbb{E}\left[\Phi\left(B_{o}\right) \Phi\left(B_{i}\right)\right] & =\mathbb{E}\left[\Phi\left(B_{i}\right) \mathbb{E}\left[\Phi\left(B_{o}\right) \mid \Phi_{B_{i}}\right]\right] \\
& =\mathbb{E}\left[\Phi\left(B_{i}\right) v(B) \lambda_{\Phi_{B_{i}}}\left(x_{o} \mid U\right)\right] \\
& =\mathbb{E}\left[\Phi\left(B_{i}\right) v(B) \mathbb{E}\left[\lambda_{\Phi_{S_{o b s}}}\left(x_{o} \mid U\right)\right] \mid \Phi_{B_{i}}\right] \\
& =v(B) \mathbb{E}\left[\mathbb{E}\left[\Phi\left(B_{i}\right) \lambda_{\Phi_{S_{o b s}}}\left(x_{o} \mid U\right)\right] \mid \Phi_{B_{i}}\right] \\
& =v(B) \mathbb{E}\left[\Phi\left(B_{i}\right) \lambda_{\Phi_{S_{o b s}}}\left(x_{o} \mid U\right)\right]
\end{aligned}
$$

which leads to $\mathbb{E}\left[\Phi\left(B_{i}\right) \lambda\left(x_{0} \mid U\right)\right]=\frac{1}{v(B)} \mathbb{E}\left[\Phi\left(B_{0}\right) \Phi\left(B_{i}\right)\right]$ as in our case the local intensity is conditional to the realization of the process $\Phi$ in $S_{o b s}$.

- for $x_{i}=x_{0}$,

$$
\begin{aligned}
\mathbb{E}\left[\Phi^{2}\left(B_{0}\right)\right] & =\mathbb{E}\left[\mathbb{E}\left[\Phi^{2}\left(B_{o}\right) \mid \Phi_{S_{o b s}}\right]\right]=\mathbb{E}\left[\Phi\left(B_{o}\right) \mathbb{E}\left[\Phi\left(B_{o}\right) \mid \Phi_{S_{o b s}}\right]\right] \\
& =\mathbb{E}\left[\Phi\left(B_{o}\right) v(B) \lambda\left(x_{o} \mid U\right)\right] \\
& =v(B) \mathbb{E}\left[\Phi\left(B_{o}\right) \lambda\left(x_{o} \mid U\right)\right]
\end{aligned}
$$

which leads to $\mathbb{E}\left[\Phi\left(B_{0}\right) \lambda\left(x_{o} \mid U\right)\right]=\frac{1}{v(B)} \mathbb{E}\left[\Phi^{2}\left(B_{0}\right)\right]$. 


\section{ARTICLE IN PRESS}

Consequently, $\widetilde{C}_{o}=\frac{1}{v(B)} C_{o}$ and we get

$$
\mu=C^{-1} C_{o}+\frac{1-\mathbf{1}^{T} C^{-1} C_{o}}{\mathbf{1}^{T} C^{-1} \mathbf{1}} C^{-1} \mathbf{1} .
$$

Interpolating $\Phi\left(B_{0}\right) / v(B)$ or $\lambda\left(x_{0} \mid U\right)$ leads to the same kriging weights.

Finally,

$$
\mathbb{E}\left[\widehat{\lambda}\left(x_{o} \mid U\right)\right]=\mathbb{E}\left[\sum_{x_{i} \in S_{o b s}} \mu_{i} \frac{\Phi\left(B_{i}\right)}{v(B)}\right]=\mathbb{E}\left[\frac{\Phi\left(B_{0}\right)}{v(B)}\right] \underset{v(B) \rightarrow 0}{\longrightarrow} \lambda\left(x_{o} \mid U\right)
$$

shows that $\widehat{\lambda}\left(x_{0} \mid U\right)$ is an asymptotically unbiased predictor of $\lambda\left(x_{0} \mid U\right)$.

\subsection{Properties of the interpolator}

In order to develop the variance of the kriging interpolator, we use the following Neumann series (see e.g. Petersen and Pedersen, 2012) to invert the covariance matrix $C$, which holds when $\lambda v(B)$ tends to 0 :

$$
C^{-1}=\frac{1}{\lambda \nu(B)}\left[\mathbf{I}+\lambda \nu(B) J_{\lambda}\right]
$$

where a generic element of the matrix $J_{\lambda}$ is given by

$$
\begin{aligned}
J_{\lambda}[i, j]= & \sum_{k=1}^{\infty}(-1)^{k} \lambda^{k-1}\left(g\left(x_{i}, x_{l_{1}}\right)-1\right)\left(g\left(x_{l_{k-1}}, x_{j}\right)-1\right) \\
& \times \int_{S_{o b s}^{k-1}} \prod_{m=1}^{k-2}\left(g\left(x_{l_{m}}, x_{l_{m+1}}\right)-1\right) \mathrm{d} x_{l_{1}} \ldots \mathrm{d} x_{l_{k-1}} .
\end{aligned}
$$

Proposition 3.2. When $\lambda v(B)$ tends to 0 , the variance of $\widehat{\lambda}\left(x_{0} \mid U\right)$ is

$$
\begin{aligned}
\operatorname{Var} & \left.\widehat{\lambda}\left(x_{o} \mid U\right)\right)=\frac{\lambda}{v(B)}+2 \lambda^{2} \mathbb{I}_{x_{0}}^{T} J \mathbb{I}_{x_{0}}+2 \lambda^{3} v(B) \mathbb{I}_{x_{0}}^{T} J_{\lambda}\left(G_{o}-1\right) \\
& +\lambda^{3} v^{2}(B)\left(G_{o}-1\right)^{T}\left(G_{o}-1\right)+\lambda^{4} v^{3}(B)\left(G_{0}-1\right)^{T} J_{\lambda}\left(G_{o}-1\right) \\
& +\frac{1-\left[1+\lambda v(B) \mathbf{1}^{T} J_{\lambda} \mathbb{I}_{x_{0}}+\lambda v(B) \mathbf{1}^{T}\left(G_{o}-1\right)+\lambda^{2} v^{2}(B) \mathbf{1}^{T} J_{\lambda}\left(G_{o}-1\right)\right]^{2}}{\frac{v\left(S_{o b s}\right)}{\lambda}+v^{2}(B) \mathbf{1}^{T} J_{\lambda} \mathbf{1}} .
\end{aligned}
$$

In estimation, i.e. when $x_{0} \in S_{o b s}$, we get the following approximation,

$$
\operatorname{Var}\left(\widehat{\lambda}\left(x_{0} \mid U\right)\right) \approx \frac{\lambda}{v(B)}
$$

In prediction, i.e. when $x_{0} \notin S_{o b s}$, the variance reduces to

$$
\begin{aligned}
\operatorname{Var}\left(\widehat{\lambda}\left(x_{o} \mid U\right)\right)= & \lambda^{3} v^{2}(B)\left(G_{o}-1\right)^{T}\left(G_{o}-1\right)+\lambda^{4} v^{3}(B)\left(G_{o}-1\right)^{T} J_{\lambda}\left(G_{o}-1\right) \\
& +\frac{1-\left[\lambda v(B) \mathbf{1}^{T}\left(G_{o}-1\right)+\lambda^{2} v^{2}(B) \mathbf{1}^{T} J_{\lambda}\left(G_{o}-1\right)\right]^{2}}{\frac{v\left(S_{o b s}\right)}{\lambda}+v^{2}(B) \mathbf{1}^{T} J_{\lambda} \mathbf{1}}
\end{aligned}
$$

Proof. From e.g. Cressie (1993), the variance of the predictor is given by

$$
\begin{aligned}
\operatorname{Var}\left(\hat{\lambda}\left(x_{o} \mid U\right)\right) & =\operatorname{Var}\left(\sum_{x_{i} \in S_{o b s}} \mu_{i} \frac{\Phi\left(B_{i}\right)}{v(B)}\right)=\frac{1}{v^{2}(B)} \mu^{T} C^{-1} \mu \\
& =\frac{1}{v^{2}(B)}\left\{C_{o}^{T} C^{-1} C_{o}+\frac{1-\left(\mathbf{1}^{T} C^{-1} C_{o}\right)^{2}}{\mathbf{1}^{T} C^{-1} \mathbf{1}}\right\} .
\end{aligned}
$$




\section{ARTICLE IN PRESS}

- When estimating the local intensity, i.e. for $x_{0}$ lying in the observation window, we have

$$
C_{o}=\lambda v(B) \mathbb{I}_{x_{o}}+\lambda^{2} v^{2}(B)\left(G_{o}-1\right) \text {. }
$$

Thus, from Eq. (8):

$$
\begin{aligned}
C_{o}^{T} C^{-1} C_{o}= & \lambda v(B)\left[1+\lambda \nu(B)\left(J_{\lambda}\left(x_{0}, x_{o}\right)+2 \mathbb{I}_{\chi_{0}}^{T}\left(G_{o}-1\right)\right)\right. \\
& \left.\lambda^{2} v^{2}(B)\left(2 \mathbb{I}_{x_{0}}^{T} J_{\lambda}\left(G_{o}-1\right)+\left(G_{o}-1\right)^{T}\left(G_{o}-1\right)\right) \lambda^{3} v^{3}(B)\left(G_{o}-1\right)^{T} J_{\lambda}\left(G_{o}-1\right)\right],
\end{aligned}
$$

where $J_{\lambda}(y, z)=\sum_{k=1}^{\infty}(-1)^{k} \lambda^{k-1} \int_{S_{o b s}^{k-1}}\left(g\left(y, x_{l_{1}}\right)-1\right) \prod_{m=1}^{k-2}\left(g\left(x_{l_{m}}, x_{l_{m+1}}\right)-1\right)\left(g\left(x_{l_{k-1}}, z\right)-\right.$ 1) $\mathrm{d} x_{l_{1}} \ldots \mathrm{d} x_{l_{k-1}}$,

$$
\mathbf{1}^{T} C^{-1} C_{o}=1+\lambda \nu(B)\left[\mathbf{1}^{T} J \lambda \mathbb{I}_{x_{o}}+\mathbf{1}^{T}\left(G_{o}-1\right)+\lambda \nu(B) \mathbf{1}^{T} J_{\lambda}\left(G_{o}-1\right)\right]
$$

and

$$
\mathbf{1}^{T} C^{-1} \mathbf{1}=\frac{1}{\lambda \nu(B)}\left[n_{o b s}+\lambda v(B) \mathbf{1}^{T} J_{\lambda} \mathbf{1}\right]=\frac{\nu\left(S_{o b s}\right)}{\lambda v^{2}(B)}+\mathbf{1}^{T} J_{\lambda} \mathbf{1}
$$

Then, if $v(B)$ is very small, $\frac{C_{0}^{T} C^{-1} C_{0}}{v^{2}(B)}$ varies in $\frac{\lambda}{v(B)}$ and $\frac{1-\left(\mathbf{1}^{T} C^{-1} C_{0}\right)^{2}}{v^{2}(B) \mathbf{1}^{T} C^{-1} \mathbf{1}}$ in $\frac{\lambda}{v\left(S_{o b s}\right)}$. Thus, we get Eq. (10).

- When predicting the local intensity, i.e. for $x_{0}$ outside the observation window, we have $C_{0}=$ $\lambda^{2} v^{2}(B)\left(G_{0}-1\right)$. Thus, from

$$
C_{o}^{T} C^{-1} C_{o}=\lambda^{3} v^{3}(B)\left(G_{o}-1\right)^{T}\left(G_{o}-1\right)+\lambda^{4} \nu^{4}(B)\left(G_{o}-1\right)^{T} J_{\lambda}\left(G_{o}-1\right)
$$

and

$$
\mathbf{1}^{T} C^{-1} C_{o}=\lambda \nu(B) \mathbf{1}^{T}\left(G_{o}-1\right)+\lambda^{2} \nu^{2}(B) \mathbf{1}^{T} J_{\lambda}\left(G_{o}-1\right)
$$

we get Eq. (11).

\subsection{Defining an optimal mesh size}

The Integrated Mean Squared Error of $\widehat{\lambda}(x \mid U)$ is defined as

$$
\operatorname{IMSE}(\widehat{\lambda}(x \mid U))=\int_{S}\left[(\lambda(x \mid U)-\mathbb{E}[\widehat{\lambda}(x \mid U)])^{2}+\operatorname{Var}(\widehat{\lambda}(x \mid U))\right] \mathrm{d} x .
$$

When estimating the local intensity, this leads to the following approximation:

$$
\operatorname{IMSE}(\widehat{\lambda}(x \mid U)) \approx \frac{\sqrt{v(B)}}{12} \int_{S_{\text {obs }}}\|\nabla \lambda(x \mid U)\|^{2} \mathrm{~d} x+\frac{\lambda \nu\left(S_{o b s}\right)}{\nu(B)} .
$$

We propose to find the optimal mesh of the estimation grid by minimizing $\operatorname{IMSE}(\widehat{\lambda}(x \mid U))$ (see Appendix C), and we get:

$$
v_{o p t}(B)=\sqrt{\frac{12 \lambda \nu\left(S_{o b s}\right)}{\int_{S_{o b s}}\|\nabla \lambda(x \mid U)\|^{2} \mathrm{~d} x}} .
$$

Note that because the optimal mesh depends on the inverse of squared $L_{2}$-norm of the gradient of the local intensity, it decreases for clustered point patterns. Conversely, it increases for regular point patterns.

In practice the optimal mesh can be approximated by estimating the gradient of the intensity over a fine grid (see Appendix D).

When predicting the local intensity, the smaller the mesh, the better. Computation time is the only limit. 


\section{ARTICLE IN PRESS}

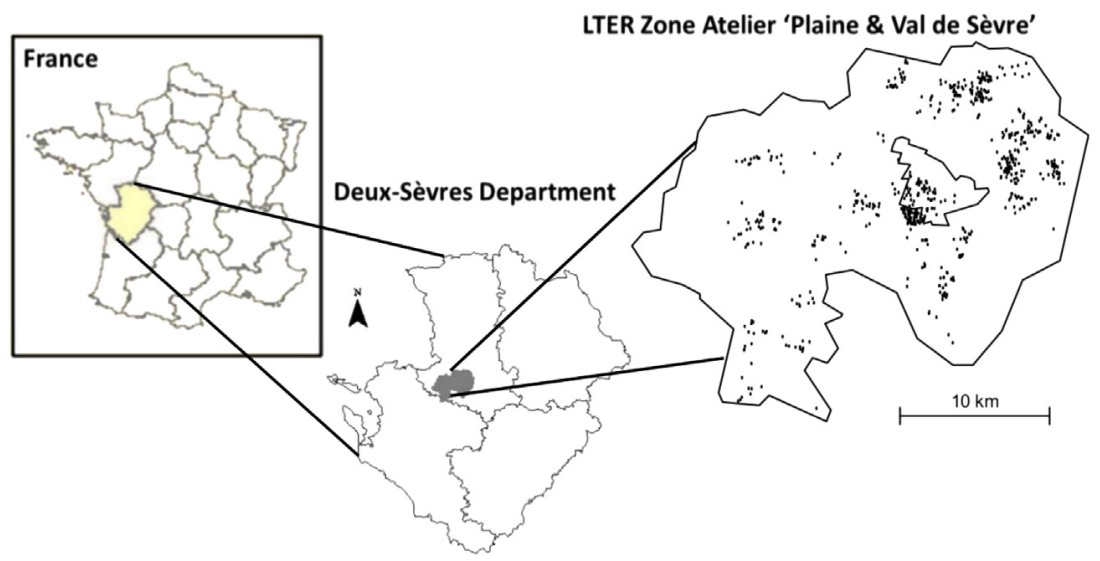

Fig. 1. Montagu's Harriers nest locations in the Zone Atelier "Plaine \& Val de Sèvre".

\section{Real case study}

In this section we estimate and predict the intensity of Montagu's Harriers' nest locations in the Zone Atelier "Plaine \& Val de Sèvre"1 (Fig. 1), a NATURA2000 site in France of $450 \mathrm{~km}^{2}$, designated for its remarkable diversity of bird species. Dots in Fig. 1 represent the exhaustive collection of Montagu's Harriers' nest locations. The area in the center of the Zone Atelier delineates the administrative boundaries of the commune Saint-Martin-de-Bernegoue, which will be used for prediction.

\subsection{Estimation of the pair correlation function}

The pair correlation is estimated as defined in Stoyan and Stoyan (1994):

$$
\widehat{g}(r)=\frac{1}{2 \pi r} \sum_{\xi \in \Phi_{S_{o b s}}} \sum_{\zeta \in \Phi_{S_{o b s}}}^{\neq} \frac{\mathbf{k}_{h}(r-\|\xi-\zeta\|)}{\operatorname{prop}\left(S_{o b s} \cap S_{o b s, \xi-\zeta}\right)}
$$

where $\mathbf{k}_{h}$ is the Epanechnikov kernel with bandwidth $h$, the optimal Stoyan's bandwidth equals to $0.15 / \sqrt{\Phi\left(S_{o b s}\right) / v\left(S_{o b s}\right)}$ and prop $\left(S_{o b s} \cap S_{o b s, \xi-\zeta}\right)$ is the proportion of translations of $(\xi, \zeta)$ which have both $\xi$ and $\zeta$ inside $S_{o b s}$. Fig. 2(a) shows the pair correlation function estimated from either all data point locations (solid line) or only the ones outside the boundaries of Saint-Martin-de-Bernegoue (dashed line). These estimates are characteristic of a Thomas cluster process with an infinite range of correlation, see Illian et al. (2008).

\subsection{Intensity estimation}

For our kriging estimator, the optimal mesh is obtained by minimizing the IMSE. Usual nonparametric estimation methods also require to preliminary set the smoothing parameter and this parameter is chosen as an optimal value minimizing a specific criterion (typically mean square error, integrated bias, asymptotic mean square error). In our case, we have an explicit formula of the optimal mesh (Eq. (13)), which depends on the unknown terms $\lambda$ and $\lambda(x \mid U)$. If $\hat{\lambda}=\Phi\left(S_{o b s}\right) / v\left(S_{o b s}\right)$ appears to be a natural candidate to estimate $\lambda$, the challenging goal is to estimate $\int_{S_{o b s}}\|\nabla \lambda(x \mid U)\|^{2} \mathrm{~d} x$. Based on simulation experiments (Appendix D), we consider a Gaussian kernel (Silverman, 1986), with a bandwidth minimizing the mean-square error criterion defined by Diggle (1985), to get a good

\footnotetext{
1 http://www.za.plainevalsevre.cnrs.fr/.
} 

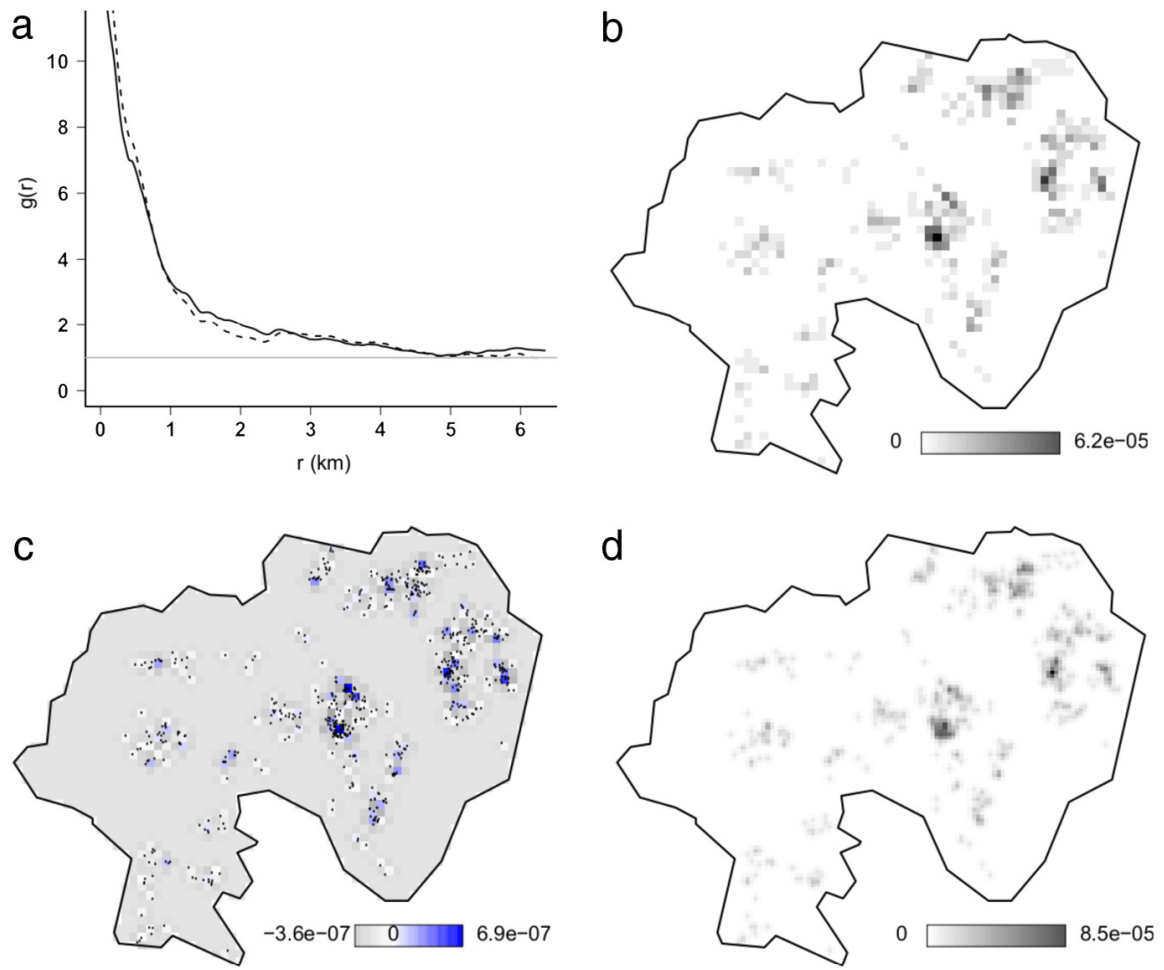

Fig. 2. (a) Estimation of the pair correlation function from all points (solid line) and without those lying in Saint-Martin-de Bernegoue (dashed line). Estimation of Montagu's Harriers nest locations using our kriging interpolator on the optimal grid (b) and using a Gaussian kernel on a $128 \times 128$ grid (d). (c) Difference between our estimator and the Gaussian kernel smoothing at the same resolution: dark gray (resp. blue) indicates higher values of the Gaussian kernel (resp. our kriging) estimates. (For interpretation of the references to color in this figure legend, the reader is referred to the web version of this article.)

approximation of the gradient of $\lambda(x \mid U)$ on a $200 \times 200$ grid. This methodology applied to the real dataset leads to a value of $v_{\text {opt }}(B)$ equals to 23.19 hectares, which corresponds to a grid of $64 \times 53$ cells.

Fig. 2(b) shows the kriging estimate on the optimal grid. Fig. 2(d) represents an estimate obtained by a Gaussian kernel, with a bandwidth selected as previously mentioned, on a $128 \times 128$ grid (default of the spatstat function 'density . ppp', Baddeley and Turner, 2005). Fig. 2(c) illustrates the difference between our estimation and the one obtained by Gaussian kernel smoothing at the same grid resolution. Our kriging interpolator gives higher values of the local intensity (in blue in Fig. 2(c)) close to aggregated observation points than the kernel estimator, while the maximum value may be higher for the later. This illustrates that our method may be particularly relevant for point patterns strongly aggregated at a small scale.

\subsection{Intensity prediction}

In order to apply our kriging predictor to the real dataset, we consider an unobserved window $S_{\text {unobs }}$ defined by the administrative boundaries of the commune Saint-Martin-de-Bernegoue in the center of the 'Zone Atelier' (Fig. 1). Thus, we remove the points in this area (red dots in Fig. 3) and use the remaining nest locations (blue dots in Fig. 3 ) to predict the local intensity within $S_{\text {unobs. }}$. We consider a grid of size $100 \times 100$ over $S$ to make the prediction. The estimated pair correlation function is plotted in Fig. 2(a) (dashed line). The result, zoomed in Fig. 3, shows that the kriging predictor is able to reproduce the second-order structure of the point process. In particular, it reproduces clusters as 


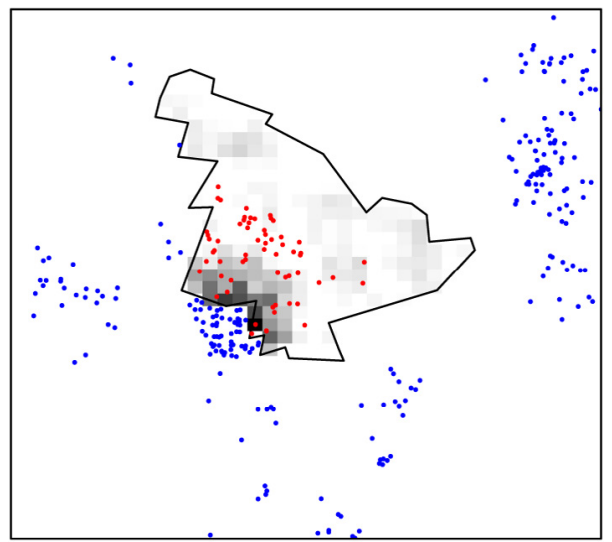

Fig. 3. Prediction within the commune of Saint-Martin-de-Bernegoue (data location is in blue; the red ones are removed for the prediction). (For interpretation of the references to color in this figure legend, the reader is referred to the web version of this article.)

soon as there are points close enough to the boundary of the unobserved area. This will be further illustrated and discussed in the next section. Note that at distances greater than the range of the pair correlation function, the method can only provide a constant intensity estimate.

\section{Illustrative simulation experiments}

\subsection{Objectives}

Now, we focus on the kriging predictor and explore its accuracy through simulation experiments, varying rate and shape of the observation window $S_{o b s}$. To measure the quality of prediction, we compute the mean bias (MB) and the mean square error of prediction (MSEP):

$$
\begin{aligned}
M B & =\frac{1}{n_{o b s}} \sum_{x \in \Phi_{S_{o b s}}} \frac{1}{n_{s i m}} \sum_{k=1}^{n_{\text {sim }}}\left(\widehat{\lambda}_{k}(x \mid U)-\lambda_{k}(x \mid U)\right), \\
M S E P & =\frac{1}{n_{o b s}} \sum_{x \in U} \frac{1}{n_{\text {sim }}} \sum_{k=1}^{n_{\text {sim }}}\left(\widehat{\lambda}_{k}(x \mid U)-\lambda_{k}(x \mid U)\right)^{2},
\end{aligned}
$$

where $\lambda_{k}$ and $\widehat{\lambda}_{k}$ correspond respectively to the intensity and its predictor on the $k$ th simulation and $n_{\text {sim }}$ is the number of simulations. We also compute the coefficient of determination $R^{2}$ of the regression between the predicted values of the local intensity and the theoretical ones.

\subsection{Experimental design}

Throughout our experimental study, in order to simplify the analysis of the two parameters of interest (rate and shape of $S_{o b s}$ ), we decide to simulate all point patterns from a single spatial point process model. We consider in the sequel a Thomas process, for which we have explicit formulas of the intensity, pair correlation function and others characteristics (see Illian et al., 2008, p. 377):

$$
\begin{aligned}
\lambda(x \mid U) & =\sum_{\xi \in \Phi_{S_{o b s}}} \frac{\mu}{2 \pi \sigma^{2}} \exp \left(-\frac{\|x-\xi\|^{2}}{2 \sigma^{2}}\right), \quad \text { for all } x \in S, \\
g(r) & =1+\frac{1}{4 \pi \kappa \sigma^{2}} \exp \left(-\frac{r^{2}}{4 \sigma^{2}}\right), \quad \text { for } r \geq 0 .
\end{aligned}
$$




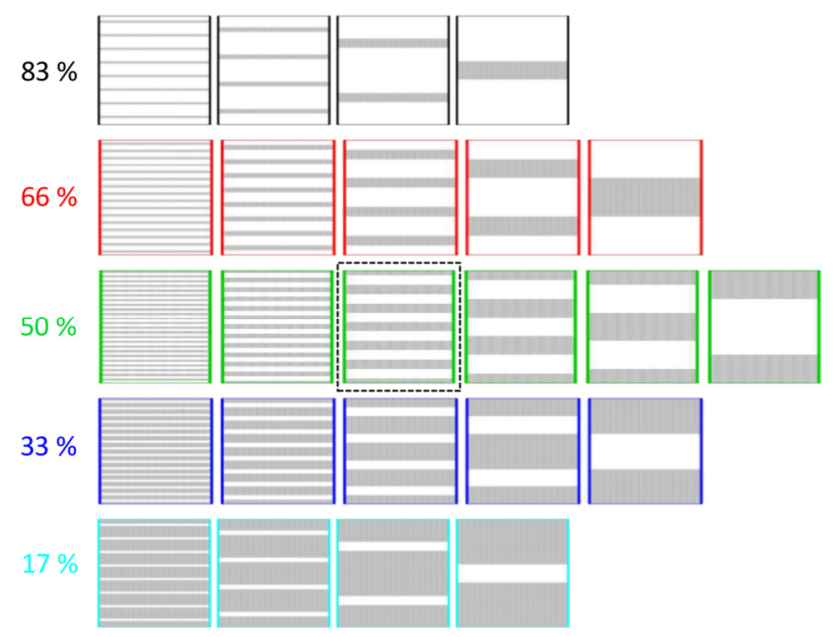

Fig. 4. Windows of interest with $S_{o b s}$ (resp. $S_{\text {unobs }}$ ) the union of white (resp. gray) bands.

Such a Cox model is of interest as it models spatial aggregation, a condition often observed in practical situations of intensity prediction. We simulate $n_{\text {sim }}=1000$ patterns of a Thomas process in the unit square with parameters:

- $\kappa=10$, the intensity of parent points from a homogeneous Poisson point process,

- $\mu=50$, the mean number of children points around each parent point from a Poisson distribution,

- $\sigma=0.05$, the standard deviation of the Gaussian density distribution centered at each parent point.

Several windows of interest $S_{i}$, with $i=1, \ldots, 24$, are considered (Fig. 4), corresponding to different observation rates (83\%, 66\%, 50\%, 33\% and $17 \%$ ). The unobserved windows $S_{\text {unobs }}$ are defined by the union of bands, with varying width (in gray in Fig. 4).

Because the weights in our kriging interpolator depend on the pair correlation function, in our experiment we compare results arising from the theoretical pair correlation function, and from its estimate defined in Section 4.1. In order to estimate the pair correlation function from similar numbers of points in each window $S_{i}$, we first simulated point patterns within a larger window (the initial one extended on the right side), so that the area of observation zones equals to one. The pair correlation function is then estimated from this first pattern and the prediction is made on its restriction to the initial unit square.

\subsection{Results}

The mean bias and the mean square error of prediction are presented in Fig. 5, with theoretical values of the pair correlation function (solid lines) or an estimate (dotted line). It shows that the mean bias has no effect on the MSEP. With theoretical values of the pair correlation function, the mean bias is close to zero whatever the width of the bands defining $S_{\text {unobs }}$, which numerically reveals the unbiasedness statistical property of our predictor. When the pair correlation function is estimated, $\lambda_{k}$ is under-estimated and the discrepancy is higher when the observation rate decreases than when the width of the unobserved bands increases.

At a given observation rate, the MSEP increases when the width of the unobserved bands increases. Indeed, the geometry of our windows of interest implies that wider the unobserved bands, less numerous they are. Consequently, for some simulated patterns, cluster points can completely fall within an unobserved band, what damages the quality of prediction. At a given value of the unobserved band, we obviously see a slight increase of the MSEP when the observation rate decreases. 


\section{ARTICLE IN PRESS}
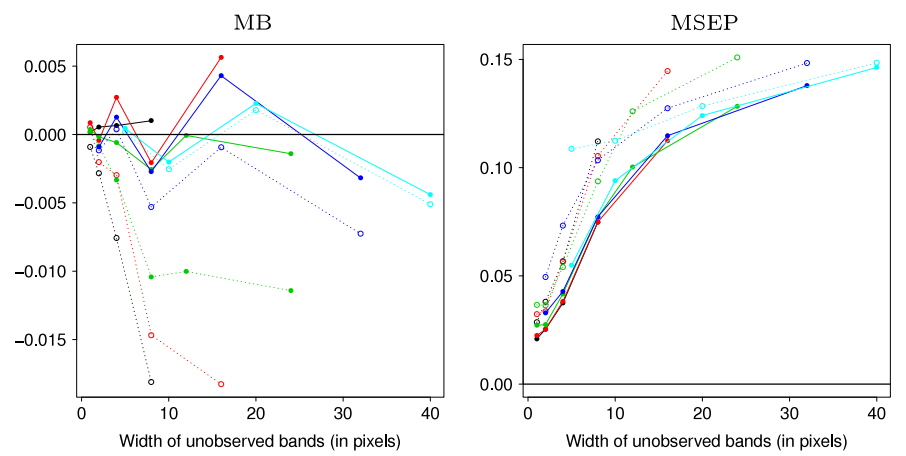

Fig. 5. Mean Bias (left) and Mean Square Error of Prediction (right) of our kriging predictor related to the width of the unobserved bands, and to the observation rates: $83 \%$ in cyan, $66 \%$ in blue, $50 \%$ in green, $33 \%$ in red and $17 \%$ in black. The lines correspond to a linear approximation of the MSEP values when $g$ is known (solid lines) or estimated (dotted lines). (For interpretation of the references to color in this figure legend, the reader is referred to the web version of this article.)

We first illustrate the influence of the estimation of the pair correlation function onto the accuracy of prediction on a single simulation. The simulated pattern, the associated theoretical intensity and the observation window, with a rate of $50 \%$ of observed areas, are represented in Fig. 6(a)-(c) respectively. The theoretical (dotted line) and estimated (solid line) pair correlation function are given in Fig. 6(d). Fig. 6(e) and (f) illustrate the theoretical local intensity in $S_{o b s}$ and the prediction in $S_{\text {unobs }}$ on a $96 \times 96$ grid, using the true (e) and the estimated (f) pair correlation function. In the first case, the prediction is relatively smooth and gives accurate results. In the second case, the prediction is more noisy, but recover the same blocks with high intensity values. In both cases, the method correctly predict the clusters when there are observations close to the unobserved bands. That is the case for all clusters located at the right hand side of the vertical line $x=0.25$. When the full cluster falls in the unobserved band, as the ones located at the left hand side of the vertical line $x=0.25$ the method fails in predicting the cluster.

We plotted (Fig. 7) the boxplot of the coefficient of determination resulting from 100 simple linear regressions between the predicted values of the local intensity and the theoretical ones, for different grid size ( $24 \times 24,48 \times 48$ and $96 \times 96)$, when the pair correlation function is estimated (Fig. 7(b)) or not (Fig. 7(a)). These results are related to an observation rate of $50 \%$, according to the window configuration highlighted Fig. 4. We obviously see that the goodness of prediction increases when the grid resolution increases and when the pair correlation function is known. We considered a $96 \times 96$ grid as it is a trade off between computation times and a small mesh, allowing a good description of the intensity variations due to clusters. We obtained, in the worst case where the pair correlation function is estimated, that the coefficient of determination $R^{2}$ is around 0.8 (median).

\section{Discussion}

Our kriging method introduced to estimate and/or predict the local intensity of a stationary and isotropic point process has a large number of advantages, particularly in prediction. Taking into account the spatial structure of the point pattern allows to perform the intensity estimation for point processes highly aggregated at fine scale. In the prediction framework, our kriging method is innovative for the interpolation of the local intensity and presents good statistical properties (unbiasedness, low variance...) when the pair correlation function is known. When it is estimated, the quality of our interpolator is slightly reduced but our results can be improved by better taking into account the double estimation of the pair correlation function and the local intensity on the same pattern.

This prediction method is less time consuming than the reconstruction methods and appears a promising way in prediction of intensity of a spatial point pattern. Note that existing prediction methods are constrained within a class of point processes (van Lieshout and Baddeley, 2001, in 

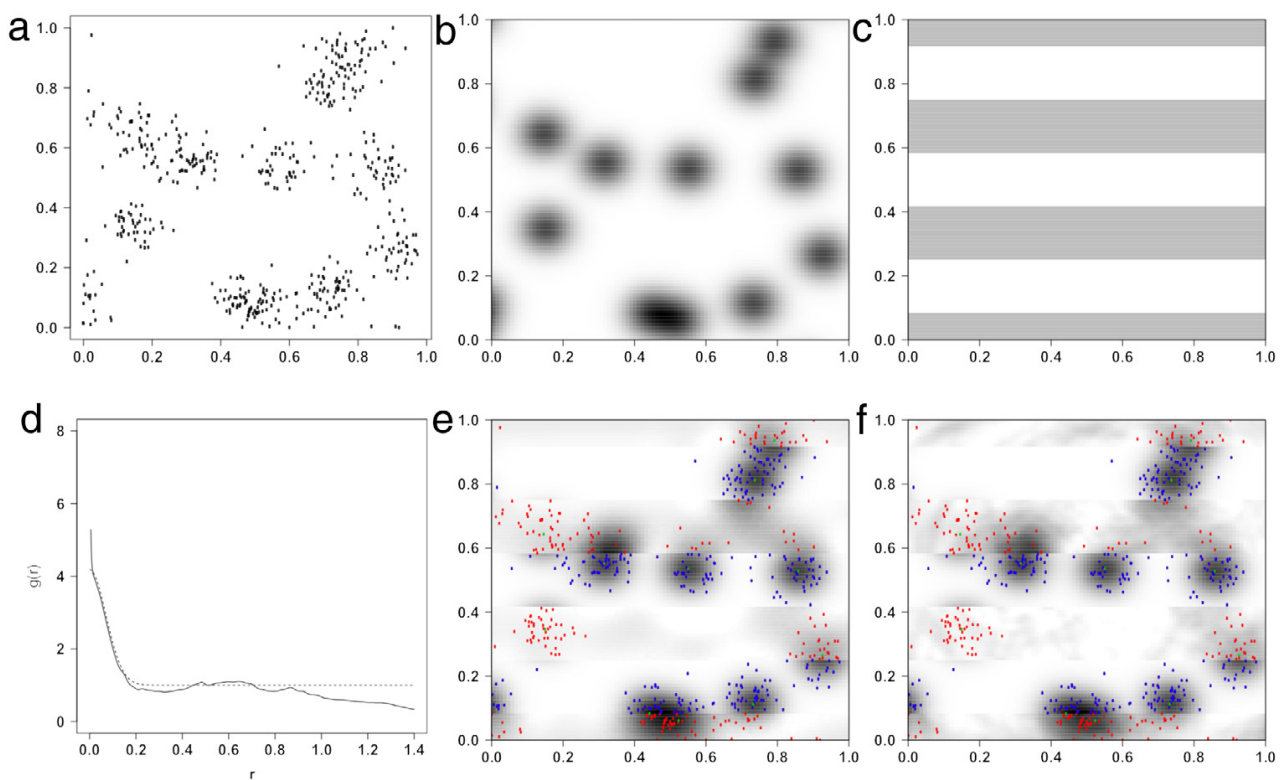

Fig. 6. (a) Simulated pattern from a Thomas process (parent points in red and children points in black). (b) Theoretical intensity from the simulated pattern. (c) Observed window (light gray) and unobserved window (red) with an observation rate of 50\%. (d) Theoretical (dotted line) and estimated (solid line) pair correlation function $g$. (e) and (f) Theoretical intensity in the observed window and predicted local intensity in the unobserved window obtained with the true pair correlation function (e) and an estimate (f). (For interpretation of the references to color in this figure legend, the reader is referred to the web version of this article.)
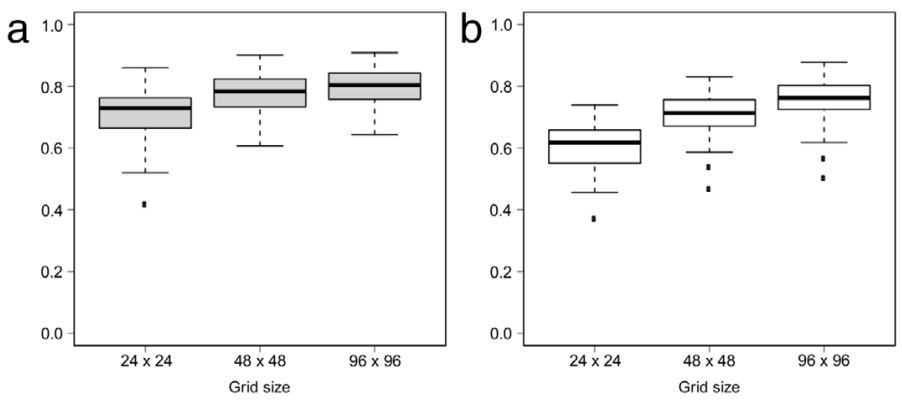

Fig. 7. $R^{2}$ in linear regressions of the predicted and theoretical values of the local intensity, associated with different grid size, when the predictions are based on the theoretical pair correlation function (a) or an estimate (b).

particular Cox processes Monestiez et al., 2006, Diggle and Ribeiro, 2007, Bellier et al., 2013, Diggle et al., 2013), making any comparison with our method very restrictive relatively to its broad scope of applications. That is for instance the case of any point process obtained by a weak dependent process (e.g. Thomas, Markov) with a parameter driven by a stationary random field at a larger scale (e.g. Cox), but not only.

Relaxing the stationary assumption implies to make further assumptions. The formalism should be quite similar to the one of this paper, but with some confounding effects as the ones observed when using the same point pattern to estimate both a spatially varying intensity and second-order characteristics (Diggle et al., 2007; Gabriel, 2014). For instance, if we consider a non stationary Cox process, we cannot disentangle the first-order non-stationarity to the second-order non-stationarity. One could thus allocate the effects at different scales. 


\section{ARTICLE IN PRESS}

In our simulations and application, we used the $\mathrm{R}$ function solve, based on the LU factorization, to compute the inverse of the covariance matrix $C$. This matrix is of dimension the square of the number of cells of the grid superimposed on the observation window. Thus, it can quickly become heavy to inverse. In such cases, estimating the matrix $\mathrm{C}^{-1}$ using Eq. (8) would be somewhat cumbersome. Thus, we propose instead to inverse the covariance matrix numerically. Several approximations could be used, depending mainly on the width of $B$ with respect to $\lambda$ and the curvature of the pair correlation function:

1. if the diameter of $B$ is large, the covariance between two tiles at distance $r$ is equal to $\lambda \nu(B) \mathbb{I}_{r=o}+$ $\lambda^{2} \int_{B \times D}(g(r+x-y)-1) \mathrm{d} x \mathrm{~d} y$. It can be approximated numerically by computing for example the integral on a fine grid. Then the finer the grid, the smaller the difference between the exact values and the approximations, but the computing time cost can become prohibitive.

2. when the diameter of $B$ becomes small, the integral can be approximated by $v(B)^{2} g(r)$ so that the covariance is approximated by $\lambda \nu(B) \mathbb{I}_{r=o}+v(B)^{2} \lambda^{2} g(r)$,

3. when the diameter becomes very small, $C$ may be approximated by $\lambda \nu(B) \mathbf{I}$, a situation seldom met in practice, since it needs a tile $B$ small enough to neglect point dependence.

Approximation (2) will thus be the most reasonable one, needing only a $B$ small enough to consider that $g(r+x)$ is almost constant for $x \in B$, but avoiding too small $B$ leading to large matrix inversion time.

Our estimation is roughly pixellated compared to kernel methods, but it does not oversmooth the intensity of highly aggregated point processes. We could take the benefit of the two approaches to get smoother estimations. Our on-going work consists in regularizing the counting process by a kernel and in defining a kriging estimator for the related random field. Our optimal grid could then be used to define an optimal bandwidth, thus eliminating the Poisson aspect of classical kernels.

Our method provides good predictions in areas at small distances of data locations. From the definition of the kriging predictor, at distances larger than the range of interaction, it only provide a constant mean value. To improve it and make it more relevant in practice, we could consider further information provided by covariates. From our application point of view, wheat field mapping could be of interest as Montagu's Harriers nest in there. From a methodological point of view, including covariates would imply that we should either consider external drift kriging (or any other universal kriging) rather than ordinary kriging; or spatial regression.

Finally, our kriging predictor depends on the count data in the grid cells, $B_{i}$, and not on exact data locations in $B_{i}$. Thus we can further consider count datasets, as it is often the case in biodiversity measures, e.g. plant species abundance. The exact position of each plant is rarely given, but we know its abundance per small unit areas. So, once the pair correlation function is estimated from the point data subset, one can apply our method to interpolate the intensity.

\section{Appendix A. Proof of Lemma 2.1}

$$
\text { (1) } \begin{aligned}
\mathbb{E}[\Phi(B) \Phi(D)] & =\mathbb{E}\left[\left(\sum_{x \in \Phi_{S}} \mathbb{I}_{B}(x)\right)\left(\sum_{y \in \Phi_{S}} \mathbb{I}_{D}(y)\right)\right] \\
& =\mathbb{E}\left[\sum_{x, y \in \Phi_{S}} \mathbb{I}_{B}(x) \mathbb{I}_{D}(y)\right] \\
& =\mathbb{E}\left[\sum_{x \in \Phi_{S}} \mathbb{I}_{B}(x) \mathbb{I}_{D}(x)\right]+\mathbb{E}\left[\sum_{x \neq y \in \Phi_{S}} \mathbb{I}_{B}(x) \mathbb{I}_{D}(y)\right] \\
& =\mathbb{E}\left[\sum_{x \in \Phi_{S}} \mathbb{I}_{B \cap D}(x)\right]+\int_{B \times D} \lambda_{2}(x, y) \mathrm{d} x \mathrm{~d} y
\end{aligned}
$$




$$
\begin{aligned}
& =\int_{B \cap D} \lambda(x) \mathrm{d} x+\lambda^{2} \int_{B \times D} g(x, y) \mathrm{d} x \mathrm{~d} y \\
& =\lambda \nu(B \cap D)+\lambda^{2} \int_{B \times D} g(x-y) \mathrm{d} x \mathrm{~d} y .
\end{aligned}
$$

The following convergence result $\mathbb{P}[\{\Phi(B)=1\} \cap\{\Phi(D)=1\}]=\lim _{v(B), v(D) \rightarrow 0} \mathbb{E}[\Phi(B) \Phi(D)]$ ends the proof.

\section{Appendix B. Proof of Proposition 2.2}

(1) $m=\mathbb{E}[Z(x)]=\mathbb{E}[\Phi(B)]=\lambda v(B)$

(2) follows from Lemma 2.1:

$$
\begin{aligned}
2 \gamma(r)= & \mathbb{E}\left[(\Phi(B)-\Phi(D))^{2}\right] \\
= & \mathbb{E}\left[(\Phi(B \backslash D)+\Phi(B \cap D)-\Phi(D \backslash B)-\Phi(D \cap B))^{2}\right] \\
= & \mathbb{E}\left[\left(\Phi\left(B_{D}\right)-\Phi\left(D_{B}\right)\right)^{2}\right] \\
= & \mathbb{E}\left[\Phi^{2}\left(B_{D}\right)\right]+\mathbb{E}\left[\Phi^{2}\left(D_{B}\right)\right]-2 \mathbb{E}\left[\Phi^{2}\left(B_{D}\right) \Phi^{2}\left(D_{B}\right)\right] \\
= & \lambda v\left(B_{D}\right)+\lambda^{2} \int_{B_{D} \times B_{D}} g(x-y) \mathrm{d} x \mathrm{~d} y+\lambda v\left(D_{B}\right)+\lambda^{2} \int_{D_{B} \times D_{B}} g(x-y) \mathrm{d} x \mathrm{~d} y \\
& -2\left(\lambda v\left(B_{D} \cap D_{B}\right)+\lambda^{2} \int_{B_{D} \times D_{B}} g(x-y) \mathrm{d} x \mathrm{~d} y\right) \\
= & \lambda\left(v\left(B_{D}\right)+v\left(D_{B}\right)\right)+\lambda^{2}\left(\int_{B_{D} \times B_{D}} g(x-y) \mathrm{d} x \mathrm{~d} y\right. \\
& \left.+\int_{D_{B} \times D_{B}} g(x-y) \mathrm{d} x \mathrm{~d} y-2 \int_{B_{D} \times D_{B}} g(x-y) \mathrm{d} x \mathrm{~d} y\right)
\end{aligned}
$$

(3) follows from the approximation $\mathbb{P}[\{\Phi(B)=1\} \cap\{\Phi(D)=1\}] \approx \lambda^{2} v(B) v(D) g(r)$ in Lemma 2.1(4).

\section{Appendix C. Proof of Eqs. (12) and (13)}

Let $B$ a square centered at 0 of area $v(B)=b^{2}$. We denote by $\nabla \lambda(x)$ the gradient vector

$$
\nabla \lambda(x)=\nabla \lambda\left(x_{1}, x_{2}\right)=\left(\partial_{1} \lambda(x), \partial_{2} \lambda(x)\right)^{T}=\left(\frac{\partial \lambda(x)}{\partial x_{1}}, \frac{\partial \lambda(x)}{\partial x_{2}}\right)^{T} .
$$

By the following Taylor expansion around the origin

$$
\lambda(x \mid U)=\lambda(0 \mid U)+x^{T} \nabla \lambda(0 \mid U)+o(\|x\|),
$$

we obtain that:

$$
\begin{aligned}
\mathbb{E}[\widehat{\lambda}(x \mid U)] & =\frac{\mathbb{E}[\Phi(B)]}{v(B)}=\frac{1}{v(B)} \int_{B} \lambda(x \mid U) \mathrm{d} x \\
& \approx \frac{1}{v(B)} \int_{B} \lambda(0 \mid U)+x^{T} \nabla \lambda(0 \mid U) \mathrm{d} x \approx \lambda(0 \mid U) \\
& \approx \lambda(x \mid U)-x^{T} \nabla \lambda(0 \mid U),
\end{aligned}
$$


and so

$$
\begin{aligned}
\int_{B}(\lambda(x \mid U)-\mathbb{E}[\widehat{\lambda}(x \mid U)])^{2} \mathrm{~d} x & \approx \int_{B}\left(x^{T} \nabla \lambda(0 \mid U)\right)^{2} \mathrm{~d} x \\
& \approx \int_{-b / 2}^{b / 2} \int_{-b / 2}^{b / 2}\left(x_{1} \partial_{1} \lambda(0 \mid U)+x_{2} \partial_{2} \lambda(0 \mid U)\right)^{2} \mathrm{~d} x_{1} \mathrm{~d} x_{2} \\
& \approx \frac{b^{4}}{12}\left[\left(\partial_{1} \lambda(0 \mid U)\right)^{2}+\left(\partial_{2} \lambda(0 \mid U)\right)^{2}\right] .
\end{aligned}
$$

By consequence when estimating the local intensity, we have from Eq. (10)

$$
\begin{aligned}
\operatorname{IMSE}(\widehat{\lambda}(x \mid U)) & \approx\left(\sum_{x_{i} \in S_{o b s}} \int_{B_{i}}(\lambda(x \mid U)-\mathbb{E}[\widehat{\lambda}(x \mid U)])^{2} \mathrm{~d} x\right)+\frac{\lambda v\left(S_{o b s}\right)}{b^{2}} \\
& \approx\left(\sum_{x_{i} \in S_{o b s}} \frac{b^{4}}{12}\left\|\nabla \lambda\left(x_{i} \mid U\right)\right\|^{2}\right)+\frac{\lambda \nu\left(S_{o b s}\right)}{b^{2}} \\
& \approx \frac{b^{4}}{12} \int_{S_{o b s}}\|\nabla \lambda(x \mid U)\|^{2} \mathrm{~d} x+\frac{\lambda v\left(S_{o b s}\right)}{b^{2}} .
\end{aligned}
$$

Deriving by the variable $b$ gives

$$
\frac{\partial \operatorname{IMSE}(\widehat{\lambda}(x \mid U))}{\partial b}=\frac{2 b}{12} \int_{S_{o b s}}\|\nabla \lambda(x \mid U)\|^{2} \mathrm{~d} x-\frac{2 \lambda v\left(S_{o b s}\right)}{b^{3}}
$$

and thus the solution of $\frac{\partial I M S E}{\partial b}=0$ is

$$
v_{o p t}(B)=\sqrt{\frac{12 \lambda \nu\left(S_{o b s}\right)}{\int_{S_{o b s}}\|\nabla \lambda(x \mid U)\|^{2} \mathrm{~d} x}}
$$

which is a minimum.

\section{Appendix D. Optimal mesh in practice}

The optimal mesh of the estimation grid, $v_{\text {opt }}(B)$, depends on the unknown terms $\lambda$ and $\nabla \lambda(x \mid U)$. In this section, we compare different methods which could be used to compute $v_{\text {opt }}(B)$ in practice. We simulated point patterns in the unit square from Thomas point processes with different set of parameters: $(\kappa, \mu) \in\{(10,50) ;(22,23) ;(50,10)\}$ and $\sigma \in\{0.001,0.0025,0.005,0.01,0.025,0.05\}$. Then, $v_{\text {opt }}(B)$ is computed as follows. First we estimate/compute the intensity on a $N \times N$ grid. Second, we deduce its gradient from the rate of change between the estimated intensity and its one-cell translated value. Third, we compute $v_{\text {opt }}(B)$ and its related grid size (in number of pixels).

We used different methods to estimate the intensity. The counting method consists in estimating the local intensity in each pixel $B_{i}$ by $\widehat{\lambda}\left(x_{i} \mid U\right)=\Phi\left(B_{i}\right) / v\left(B_{i}\right)$. The global kernel smoothing method is based on a Gaussian kernel estimator with global bandwidth and without border correction, so $\widehat{\lambda}(x \mid U)=\sum_{\xi \in \Phi} h^{-2} w(\|x-\xi\| / h)$, where $w$ is the density function of a standard normal distribution. The $k$-nearest neighbors method is an adaptive nonparametric estimation so that $\widehat{\lambda}(x \mid U)=1 /\left(\pi d_{k}(x)^{2}\right)$, with $d_{k}(x)$ the distance of $x$ to its $k$-nearest neighbor. Note that we also compute the theoretical value of the intensity from Eq. (14).

We considered three $N \times N$-grids, with $N \in\{100,200,500\}$. We compared the distributions of the optimal grid sizes obtained from 100 realizations of each process and from the different methods, to the theoretical ones. We select the method which provides the more accurate results and the less sensitivity to the different scenario. If the counting method works well when the pattern is strongly aggregated at very small scale $(\sigma \leq 0.005)$, it requires a fine grid and is inaccurate for other scales of clustering. Thus in the following it will be no longer considered. The other methods provide globally 


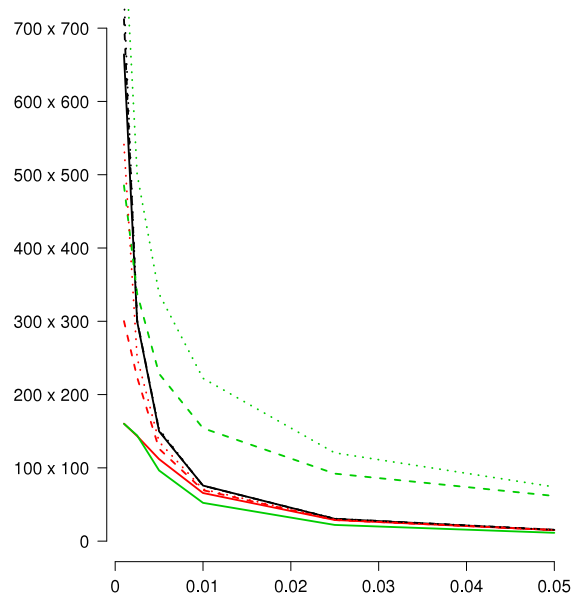

Fig. D.8. Mean of optimal grid sizes computed from 100 Thomas processes with parameters $\kappa=10, \mu=50$ and from the $k$-nearest neighbor based method (green), the kernel based method (red), both evaluated on a $100 \times 100$ grid (solid line), a $200 \times 200$ grid (dashed line) and a $500 \times 500$ grid (dotted line), compared to the theoretical value (black). (For interpretation of the references to color in this figure legend, the reader is referred to the web version of this article.)

much better values of the optimal grid size. While $\lambda(x \mid U)$ may be roughly estimated, the integral of its gradient is sufficiently well approximated to obtain good results. Fig. D.8 shows the optimal grid sizes (in number of pixels) obtained from different size of the estimation grid: $100 \times 100$ (solid line), $200 \times 200$ (dashed line) and $500 \times 500$ (dotted line). The theoretical grid size is in black and the ones derived from the kernel smoothing and the $k$-nearest neighbors method are in red and green respectively. This figure is related to the Thomas process with parameters $\kappa=10, \mu=50$, and we get similar results from the other set of parameters. It appears that the $k$-nearest neighbors based method is very sensitive to the size of the estimation grid and tends to over-estimate the optimal grid size. The kernel based method under-estimates the optimal grid size when the estimation grid is not fine enough and when the scale of clustering in very small.

From this simulation study, we recommend the kernel based method on a $200 \times 200$ grid to first estimate $\nabla \lambda(x \mid U)$ and then compute the optimal mesh or equivalently the optimal grid size.

\section{References}

Baddeley, A., Turner, R., 2005. Spatstat: an R package for analyzing spatial point patterns. J. Stat. Softw. 12 (6), 142.

Bellier, E., Monestiez, P., Certain, G., Chadœuf, J., Bretagnolle, V., 2013. Reducing the uncertainty of wildlife population abundance: model-based versus design-based estimates. Environmetrics 24 (7), 476-488.

Chilès, J., Delfiner, P., 2012. Geostatistics: Modeling Spatial Uncertainty, second ed. John Wiley \& Sons, New York.

Chiu, S., Stoyan, D., Kendall, W., Mecke, J., 2013. Stochastic Geometry and Its Applications, third ed. John Wiley \& Sons, New York.

Cressie, N., 1993. Statistics for Spatial Data, revised ed. John Wiley \& Sons, New York.

Devroye, L., 1989. The double kernel method in density estimation. Les Ann. de l'I.H.P. Sect. B 25 (4), 533-580.

Diggle, P., 1985. A kernel method for smoothing point process data. Appl. Stat. 34, 138-147.

Diggle, P., Gómez-Rubio, V., Brown, P., Chetwynd, A., Gooding, S., 2007. Second-order analysis of inhomogeneous spatial point processes using case-control data. Biometrics 63 (2), 550-557.

Diggle, P., Moraga, P., Rowlingson, B., Taylor, B., 2013. Spatial and spatio-temporal log-gaussian Cox processes: Extending the geostatistical paradigm. Statist. Sci. 28 (4), 542-563.

Diggle, P., Ribeiro, P., 2007. Model-Based Geostatistics. Springer, New York.

Gabriel, E., 2014. Estimating second-order characteristics of inhomogeneous spatio-temporal point processes: influence of edge correction methods and intensity estimates. Methodol. Comput. Appl. Probab. 16 (2), 411-431.

Guan, Y., 2008. On consistent nonparametric intensity estimation for inhomogeneous spatial point processes. J. Amer. Statist. Assoc. 103 (483), 1238-1247.

Härdle, W., 1991. Smoothing Techniques, with Implementation in S. Springer \& Verlag, New York.

Illian, J., Penttinen, A., Stoyan, H., Stoyan, D., 2008. Statistical Analysis and Modelling of Spatial Point Patterns. John Wiley \& Sons, London.

Matheron, G., 1962. Traité de Géostatistique Appliquée: Mémoires du Bureau de Recherches Géologiques et Minières, Tome I, No. 14. Editions Technip, Paris. 
Matheron, G., 1963. Traité de Géostatistique Appliquée: Le krigeage, Tome II, No. 24. Editions BRGM, Paris.

Monestiez, P., Dubroca, L., Bonnin, E., Durbec, J., Guinet, C., 2006. Geostatistical modelling of spatial distribution of balaenoptera physalus in the northwestern mediterranean sea from sparse count data and heterogeneous observation efforts. Ecol. Modell. 193, 615-628.

Petersen, K., Pedersen, M., 2012. The Matrix Cookbook. Technical University of Denmark.

Silverman, B., 1986. Density Estimation for Statistics and Data Analysis. Chapman \& Hall/CRC, London.

Stoyan, D., Stoyan, H., 1994. Fractals, Random Shapes and Point Fields: Methods of Geometrical Statistics. John Wiley \& Son. Tscheschel, A., Stoyan, D., 2006. Statistical reconstruction of random point patterns. Comput. Statist. Data Anal. 51, 859-871.

van Lieshout, M.-C., 2012. Estimation of the intensity function of a point process. Methodol. Comput. Appl. Probab. 14, 567-578 van Lieshout, M.-C., Baddeley, A., 2001. Extrapolating and interpolating spatial patterns. In: Lawson, A.B., Denison, D.G.T. (Eds.), In Spatial Cluster Modelling. Chapman and Hall/CRC, Press, Boca Raton, pp. 61-86.

Wackernagel, H., 2003. Multivariate Geostatistics: An Introduction with Applications, third ed. Springer-Verlag.

Zhang, J., Atkinson, P., Goodchild, M., 2014. Scale in Spatial Information and Analysis. Taylor \& Francis. 